\title{
REFERENCES
}

1. C. W. Curtis and I. Reiner, Representation theory of finite groups and associative algebras, Interscience, New York, 1962.

2. J. A. Green, A transfer theorem for modular representations, J. of Algebra 1 (1964), 73-84.

3. M. F. O'Reilly, On the semisimplicity of the modular representation algebra of a finite group, Illinois J. Math. 9 (1965), 261-276.

4. I. Reiner, The integral representation ring of a finite group, Michigan Math. J. 12 (1965), 11-22.

UNIVERSITY OF ILLINOIS

\section{NOTE ON ANALYTICALLY UNRAMIFIED SEMI-LOCAL RINGS}

\author{
LOUIS J. RATLIFF, JR. ${ }^{1}$
}

All rings in this paper are assumed to be commutative rings with a unit element. If $B$ is an ideal in a ring $R$, the integral closure $B_{a}$ of $B$ is the set of elements $x$ in $R$ such that $x$ satisfies an equation of the form $x^{n}+b_{1} x^{n-1}+\cdots+b_{n}=0$, where $b_{i} \in B^{i}(i=1, \cdots, n)$. An ideal $B$ in $R$ is semi-prime in case $B$ is an intersection of prime ideals. If $R$ is an integral domain, then $R$ is normal in case $R$ is integrally closed in its quotient field. If $R$ is a semi-local (Noetherian) ring, then $R$ is analytically unramified in case the completion of $R$ (with respect to the powers of the Jacobson radical of $R$ ) contains no nonzero nilpotent elements.

Let $R$ be a semi-local ring with Jacobson radical $J$, and let $R^{*}$ be the completion of $R$. In [2], Zariski proved that if $R$ is a normal local integral domain, and if there is a nonzero element $x$ in $J$ such that $p R^{*}$ is semi-prime, for every prime divisor $\mathfrak{p}$ of $x R$, then $R$ is analytically unramified. In $[1$, p. 132] Nagata proved that if $R$ is a semilocal integral domain, and if there is a nonzero element $x$ in $J$ such that, for every prime divisor $\mathfrak{p}$ of $x R, \mathfrak{p} R^{*}$ is semi-prime and $R_{\mathfrak{p}}$ is a valuation ring, then $R$ is analytically unramified. (The condition $R_{\mathfrak{p}}$ is a valuation ring holds if $R$ is normal.) The main purpose of this note is to extend Nagata's result to the case where $R$ is a semi-local ring (Theorem 1). This extension will be given after first proving a

Received by the editors March 29, 1965.

1 Work on this paper was supported in part by the National Science Foundation. Grant GP3595. 
number of lemmas. Among these preliminary results, Lemma 3 gives a necessary and sufficient condition for $R_{\mathfrak{p}}$ to be a discrete Archimedian valuation ring (where $R$ is a Noetherian ring and $\mathfrak{p}$ is a prime divisor of a nonzero-divisor $b \in R$ ), Corollary 2 of Lemma 5 gives a sufficient condition for a Noetherian ring to be a direct sum of normal Noetherian domains, and Lemma 6 gives a characterization of analytically unramified semi-local rings.

In Lemmas 1-4 below, $R$ is a Noetherian ring, $S$ is the integral closure of $R$ in its total quotient ring, $b$ is a nonunit in $R$ which is not a divisor of zero, $\mathfrak{p}$ is a prime divisor of $b R$, and $q$ is the isolated component of zero determined by $\mathfrak{p}$. If $B$ is an ideal in $R$, then $B^{\prime} R_{\mathfrak{p}}$ is the ideal generated by $(B+\mathfrak{q}) / \mathfrak{q}$ in $R_{\mathfrak{p}}$. Likewise, if $c \in R$, then $c^{\prime}$ is the q-residue of $c$.

Lемма 1. $(b R)_{a}=b S \cap R$, and an element $c$ in $R$ is in $(b R)_{a}$ if and only if $c / b \in S$.

Proof. If $c \in(b R)_{a}$, then $c^{n}+b_{1} c^{n-1}+\cdots+b_{n}=0$, where $b_{i} \in b^{i} R$. Dividing this equation by $b^{n}$ shows that $c / b \in S$, so $c \in b S \cap R$, hence $(b R)_{a} \subseteq b S \cap R$. If $c \in b S \cap R$, then $c / b \in S$, so $(c / b)^{n}+r_{1}(c / b)^{n-1}+\cdots$ $+r_{n}=0$, where $r_{i} \in R$. Multiplying this equation by $b^{n}$ shows that $c \in(b R)_{a}$, since $c \in R$. Therefore $b S \cap R \subseteq(b R)_{a}$, hence $(b R)_{a}=b S \cap R$, q.e.d.

LEMMA 2. $R_{\mathfrak{p}}$ is a discrete Archimedian valuation ring if and only if $R_{\mathfrak{p}}$ is normal.

Proof. If $R_{\mathfrak{p}}$ is a valuation ring, then $R_{\mathfrak{p}}$ is normal. Conversely, if $R_{\mathfrak{p}}$ is normal, then $R_{\mathfrak{p}}$ is a normal local integral domain (hence, the kernel of the natural homomorphism from $R$ into $R_{\mathfrak{p}}$, which is $q$, is a prime ideal), and $\mathfrak{p}^{\prime} R_{\mathfrak{p}}$ is a prime divisor of $b^{\prime} R_{\mathfrak{p}}$. Since $b^{\prime} R_{\mathfrak{p}} \neq(0)$, height $\mathfrak{p}^{\prime} R_{\mathfrak{p}}=1$ hence $R_{\mathfrak{p}}$ is a discrete Archimedian valuation ring [3, pp. 276-278], q.e.d.

An element $c \in R$ such that $b R: c R=\mathfrak{p}$ is used in the next lemma. Such an element can be found as follows. Let $\mathfrak{p}=\mathfrak{p}_{1}, \mathfrak{p}_{2}, \cdots, \mathfrak{p}_{n}$ be the prime divisors of $b R$, and let $d$ be an element in the $\mathfrak{p}_{i}$-primary component of $b R(i=2, \cdots, n)$ which is not in $b R$. If $b R: d R \neq \mathfrak{p}$, let $e$ be an element in $(b R: d R): \mathfrak{p} R$ which is not in $b R: d R$, and let $c=d e$.

LEMмA 3. Let $c$ be an element in $R$ such that $b R: c R=\mathfrak{p} . R_{\mathfrak{p}}$ is normal if and only if $c / b \notin S$.

Proof. Let $R_{\mathfrak{p}}$ be normal. Since $b R: c R=\mathfrak{p}, b^{\prime} R_{\mathfrak{p}}: c^{\prime} R_{\mathfrak{p}}=\mathfrak{p}^{\prime} R_{\mathfrak{p}}$. Therefore $c^{\prime} \notin b^{\prime} R_{\mathfrak{p}}$, so $c^{\prime} / b^{\prime} \notin R_{\mathfrak{p}}$. Hence, since $S_{R \sim \mathfrak{p}}$ is contained in the integral closure of $R_{\mathfrak{p}}$ in its quotient field, $c / b \notin S$. Conversely, 
assume $c / b \notin S$. Since $c \mathfrak{p} \subseteq b R,(c / b) \mathfrak{p} \subseteq R$. If $(c / b) \mathfrak{p} \subseteq \mathfrak{p}$, then $b R[c / b]$ $\subseteq \mathfrak{p} R[c / b] \subseteq R$, so $R[c / b]$ is contained in the finite $R$-module $(1 / b) R$, hence $c / b \in S$. This is a contradiction, so $c p \nsubseteq b p$. Therefore, there are elements $d \in \mathfrak{p}$, and $x \in R, \notin \mathfrak{p}$, such that $c d=b x$. Then $b^{\prime} R_{\mathfrak{p}}=b^{\prime} x^{\prime} R_{\mathfrak{p}}$ $=c^{\prime} d^{\prime} R_{\mathfrak{p}} \subseteq c^{\prime} \mathfrak{p}^{\prime} R_{\mathfrak{p}} \subseteq b^{\prime} R_{\mathfrak{p}}$, so $c^{\prime} \mathfrak{p}^{\prime} R_{\mathfrak{p}}=b^{\prime} R_{\mathfrak{p}}=c^{\prime} d^{\prime} R_{\mathfrak{p}}$. Now $c^{\prime}$ is not a divisor of zero in $R_{\mathfrak{p}}$ (since $b^{\prime} x^{\prime}$ is not), so $\mathfrak{p}^{\prime} R_{\mathfrak{p}}=\left(b^{\prime} / c^{\prime}\right) R_{\mathfrak{p}}$, hence $R_{\mathfrak{p}}$ is normal (Lemma 2 , and [3, p. 277]), q.e.d.

LEMMA $4 .(b R)_{a}=b R$ if and only if $R_{\mathfrak{p}}$ is normal, for every prime divisor $p$ of $b R$.

ProOF. If $R_{\mathfrak{p}}$ is normal, for every prime divisor $\mathfrak{p}$ of $b R$, then $R_{\mathfrak{p}}$ $=S_{R \sim \mathfrak{p}}$, so $\mathfrak{p}^{\prime} R_{\mathfrak{p}} \cap S$ is a prime divisor of $b S$. Let $\mathfrak{p}_{1}, \cdots, \mathfrak{p}_{n}$ be the prime divisors of $b R$, and let $b_{i}$ be the image of $b$ in $R_{\mathfrak{p}_{\mathfrak{i}}}$. Then $(b R)_{a}$ $=b S \cap R \quad($ Lemma 1$) \subseteq\left(\cap_{1}^{n}\left(b_{i} R_{\mathfrak{p}_{\mathfrak{i}}} \cap S\right)\right) \cap R=\cap_{1}^{n}\left(b_{i} R_{\mathfrak{p}_{\mathfrak{i}}} \cap R\right)$ $=b R \subseteq(b R)_{a}$, hence $(b R)_{a}=b R$. Conversely, let $(b R)_{a}=b R$, let $\mathfrak{p}$ be a prime divisor of $b R$, and let $c$ be an element in $R$ such that $b R: c R=p$. Then $c / b \notin R$. If $R_{\mathfrak{p}}$ is not normal, then $c / b \in S$ (Lemma 3 ), hence $c \in b S \cap R=(b R)_{a}$ (Lemma 1). Since $(b R)_{a}=b R$, this is a contradiction to $c / b \notin R$. Therefore $R_{\mathfrak{p}}$ is normal, q.e.d.

Lemma 5. Let $R$ be a Noetherian ring with Jacobson radical $J$, let $b$ be a nonzero element in $J$, and let $\mathfrak{p}_{1}, \cdots, \mathfrak{p}_{n}$ be the prime divisors of $b R$. If $R_{\mathfrak{p}_{\mathfrak{i}}}$ is a discrete Archimedian valuation ring $(i=1, \cdots, n)$, then the isolated component of zero contained in $\mathfrak{p}_{i}$ is a prime ideal $q_{i}$ and $\cap_{1}^{n} q_{i}=(0)$. Moreover, $b$ is not a zero-divisor in $R$, and $(b R)_{a}=b R$.

Proof. If $R_{\mathfrak{p}_{i}}$ is a discrete Archimedian valuation ring, then $R_{\mathfrak{p}_{i}}$ is an integral domain which is not a field, so the isolated component of zero contained in $\mathfrak{p}_{i}$ is a prime ideal $\mathfrak{q}_{i}$. Since $\mathfrak{q}_{i}$ is the kernel of the natural homomorphism from $R$ into $R_{\mathfrak{p}_{i}}, q_{i}$ is contained in every $\mathfrak{p}_{i^{-}}$ primary ideal. Hence, since $b R=\bigcap_{1}^{n}\left(b_{i} R_{\mathfrak{p}_{i}} \cap R\right)$, where $b_{i}$ is the $\mathfrak{q}_{i^{-}}$ residue of $b$, and since each $p_{i}$ is a minimal prime divisor of $b R$, $Z=\bigcap_{1}^{n} q_{i} \subseteq b R$. Since $b \notin q_{i}(i=1, \cdots, n), Z: b R=Z$. This implies $Z=b R \cap(Z: b R)=b(Z: b R)$. Therefore, since $b \in J, Z=b(Z: b R)$ $=b Z \subseteq J Z \subseteq Z$. Hence, $Z=\bigcap_{1}^{\infty} J^{h} Z \subseteq \bigcap_{1}^{\infty} J^{h}=(0)$. Thus $b$ is not a zerodivisor, so $(b R)_{a}=b R$ (Lemma 4), q.e.d.

COROLlary 1. With the same $R$ and $J$ of Lemma 5, suppose there is a nonzero nilpotent element in $R$. If $b$ is a nonzero divisor in $J$, then $(b R)_{a} \neq b R$.

PROOF. If $b$ is a nonzero-divisor in $J$ such that $(b R)_{a}=b R$, then $R_{\mathrm{p}}$ is a discrete Archimedian valuation ring, for every prime divisor $p$ of $b R$ (Lemma 4). Hence by Lemma 5 , the zero ideal in $R$ is semiprime, q.e.d. 
Corollary 4 below is the next result which is needed to prove Theorem 1 , and it can be proved as a corollary to Lemma 5 . Corollaries 1,2 , and 3, and Lemma 6 are not used in the proof of Theorem 1 . They are included at this point because they are of some interest in themselves.

Corollary 2. Let $R$ be an integrally closed Noetherian ring, let $J$ be the Jacobson radical of $R$, and let $\mathfrak{q}_{1}, \cdots, \mathfrak{q}_{n}$ be the minimal prime divisors of zero. If there is a nonzero-divisor $b$ in $J$, then $R=\oplus_{1}^{n} R / q_{i}$, and $R / \mathfrak{q}_{i}$ is a normal Noetherian domain.

Proof. If $b$ is a nonzero-divisor in $J$, then $(b R)_{a}=b R$, since $R$ is integrally closed. Therefore by Corollary 1 the zero ideal in $R$ is semi-prime, and consequently the total quotient ring $Q$ of $R$ is the direct sum of $n$ fields. Since the idempotents in $Q$ are integrally dependent on $R$, they are in $R$. This, and the fact that $R$ is integrally closed, immediately imply the conclusions, q.e.d.

In Corollaries 3 and 4 and Lemmas 6 and $7, R$ is a semi-local ring with maximal ideals $M_{1}, \cdots, M_{d}, J=\bigcap_{1}^{d} M_{i}$, and $R^{*}$ is the completion of $R$.

Corollary 3. Assume that no $M_{i}$ is a prime divisor of zero, and that $R^{*}$ is integrally closed. Then the completion of each $R_{M_{i}}$ is normal (hence $R_{M_{i}}$ is a normal local domain).

Proof. Since no $M_{i}$ is a prime divisor of zero, there is a nonzerodivisor $b$ in the Jacobson radical of $R^{*}[4$, p. 267]. Hence by Corollary $2, R^{*}=\oplus R^{*} / \mathfrak{q}_{i}$, where $\mathfrak{q}_{i}$ runs through the prime divisors of zero in $R^{*}$. Since the idempotents of the total quotient ring of $R^{*}$ are in $R^{*}$, no maximal ideal in $R^{*}$ contains more than one primed divisor of zero. Therefore, there are $d$ prime divisors of zero in $R^{*}$, since $R^{*} / \mathfrak{q}_{i}$ is a complete normal local domain. Let $M_{i} R^{*}$ be the maximal ideal in $R^{*}$ which contains $q_{i}$. Then it is immediately seen that $R_{M_{i} R^{*}}^{*}=R^{*} / \mathfrak{q}_{i} \supseteq R /\left(\mathfrak{q}_{i} \cap R\right)=R_{M_{i}}$. Since $R_{M_{i}}$ is a dense subspace of $R^{*} / \mathfrak{q}_{i}\left[4\right.$, p. 283], the completion of $R_{M_{i}}$ is normal. It is well known $\left[1\right.$, p. 59] that this implies that $R_{M_{i}}$ is a normal local domain, q.e.d.

LEMMA 6. Let $b$ be a nonzero-divisor in $J$, let $R^{* \prime}$ be the integral closure of $R^{*}$ in its total quotient ring, and let $T=R^{* \prime} \cap R^{*}[1 / b]$. If there is an integer $n$ such that $b^{n} T \subseteq b R^{*}$, then $R$ is analytically unramified. Conversely, if $R$ is analytically unramified, then for every nonzerodivisor $c$ in $R$ there is an integer $k$ (depending on $c$ ) such that $c^{k}\left(R^{* \prime} \cap R^{*}[1 / c]\right) \subseteq c R^{*}$.

Proof. Since $b$ is not a divisor of zero in $R, b$ is not a divisor of zero in $R^{*}\left[4\right.$, p. 267], so $R^{*}[1 / b]$ is contained in the total quotient 
ring $Q$ of $R^{*}$. Let $x$ be a nilpotent element in $R^{*}$. Then $x / b^{i} \in T$, for all $i \geqq 1$. Therefore, if $b^{n} T \subseteq b R^{*}$, then $x \in b^{i} T \subseteq b^{i-n+1} R^{*} \subseteq J^{i-n+1} R^{*}$, for all $i \geqq n$. Since $\cap J^{i} R^{*}=0, x=0$. Hence $R$ is analytically unramified. Conversely, let $R$ be analytically unramified and let $\mathfrak{q}_{1}, \cdots, \mathfrak{q}_{n}$ be the prime divisors of zero in $R^{*}$. Then $R^{* \prime}=\bigoplus_{1}^{n}\left(R^{*} / \mathfrak{q}_{i}\right)^{\prime}$, where $\left(R^{*} / \mathfrak{q}_{i}\right)^{\prime}$ is the integral closure of $R^{*} / \mathfrak{q}_{i}$. Since $\left(R^{*} / \mathfrak{q}_{i}\right)^{\prime}$ is a finite $R^{*} / \mathfrak{q}_{i}$ module [1, p. 112], $R^{* \prime}$ is a finite $R^{*}$-module. Thus $R^{* \prime} \cap R^{*}[1 / c]$ is a finite $R^{*}$-module, for every non-zero-divisor $c$ in $R$. Hence, since every element in $R^{* \prime} \cap R^{*}[1 / c]$ can be written in the form $r / c^{i}$, where $r \in\left(c^{i} R^{*}\right)_{a}$, the last statement is clear, q.e.d.

Corollary 4. With the same notation as Lemma 6 , assume $\left(b R^{*}\right)_{a}$ $=b R^{*}$. Then $R$ is analytically unramified.

Prook. If $t \in T$, then $t=r / b^{i}$, where $r \in\left(b^{i} R^{*}\right)_{a}$. Since $b R^{*}$ and $b^{i} R^{*}$ have the same prime divisors, $\left(b^{i} R^{*}\right)_{a}=b^{i} R^{*}$ (Lemma 4). Therefore $T=R^{*}$, hence $b T=b R^{*}$, and so $R$ is analytically unramified by Lemma 6, q.e.d.

LEMMA 7. Let $\mathfrak{p}$ be a height one prime ideal in $R$. If $R_{\mathfrak{p}}$ is normal, and if $\mathfrak{p} R^{*}=\bigcap_{1}^{h} p_{i}^{*}$, where each $\mathfrak{p}_{i}^{*}$ is a prime ideal in $R^{*}$, then each $R_{\mathfrak{p}_{i}^{*}}^{*}$ is normal, and $\mathfrak{p}^{(n)} R^{*}=\bigcap_{1}^{h} \mathfrak{p}_{i}^{*(n)}$ (where $\mathfrak{q}^{(n)}$ is the nth symbolic power of a prime ideal q).

Proof. Since $R_{\mathfrak{p}}$ is a normal local domain which is not a field, $\mathfrak{p}$ is not a prime divisor of zero. Let $b$ be an element in $\mathfrak{p}$ such that $b^{\prime} R_{\mathfrak{p}}$ $=\mathfrak{p}^{\prime} R_{\mathfrak{p}}\left(B^{\prime} R_{\mathfrak{p}}\right.$ denotes the ideal in $R_{\mathfrak{p}}$ generated by an ideal $B$ in $\left.R\right)$. Then $0: b R \subseteq q$, where $\mathfrak{q}$ is the prime divisor of zero contained in $\mathfrak{p}$. Therefore, $(0: b R) R^{*}=0 R^{*}: b R^{*}\left[4\right.$, p. 267] $\subseteq \mathfrak{q} R^{*} \subset \mathfrak{p} R^{*} \subseteq \mathfrak{p}_{i}^{*}$ $(i=1, \cdots, h)$. Fix $i$, set $\mathfrak{p}_{i}^{*}=p^{*}$, and let $q^{*}$ be a prime divisor of $0 R^{*}$ which is contained in $p_{i}^{*}$. Then $q^{*} \cap R$ is a prime divisor of zero $\left[4\right.$, p. 267] and is contained in $\mathfrak{p}=\mathfrak{p}^{*} \cap R$. Hence $\mathfrak{q}^{*} \cap R=\mathfrak{q}$. Further, since $q$ is the only $q$-primary ideal, every $q^{*}$-primary ideal contracts in $R$ to $\mathfrak{q}$. Hence $R_{\mathfrak{p}}$ is a subring of $R_{\mathfrak{p}^{*}}^{*}$, and, since $0 R^{*}: b R^{*} \subseteq \mathfrak{q} R^{*}, b^{\prime}$ is not a zero-divisor in $R_{\mathfrak{p}^{*}}^{*}$. Since $\mathfrak{p} R^{*}$ is semi-prime, $b^{\prime} R_{\mathfrak{p}^{*}}^{*}=\mathfrak{p}^{\prime} R_{\mathfrak{p}}^{*}$ $=\mathfrak{p}^{* \prime} R_{\mathfrak{p}^{*}}^{*}$. Therefore $R_{\mathfrak{p}^{*}}^{*}$ is normal (Lemma 2 and [3, pp. 276-278]). The proof that $\mathfrak{p}^{(n)} R^{*}=\bigcap_{1}^{h} \mathfrak{p}_{i}^{*(n)}$ is the same as that in [2]. Namely, since the result is true for $n=1$, let $n>1$ and assume $\mathfrak{p}^{(n-1)} R^{*}$ $=\bigcap_{1}^{h} \mathfrak{p}_{i}^{*(n-1)}$. Let $c$ be an element in $b R: \mathfrak{p}$ which is not in $\mathfrak{p}$ (since $\left.b^{\prime} R_{\mathfrak{p}}=\mathfrak{p}^{\prime} R_{\mathfrak{p}}, b R: \mathfrak{p} \Phi \mathfrak{p}\right)$, and let $d^{*} \in \bigcap_{1}^{h} \mathfrak{p}_{i}^{*(n)} \subset \mathfrak{p} R^{*}$. Since $c \in b R^{*}: \mathfrak{p} R^{*}$, $c d^{*}=b r^{*}$, for some $r^{*} \in R^{*}$, hence by the choice of $c$ and $b, b^{\prime} r^{* \prime} R_{\mathfrak{p}_{i}^{*}}^{*}$ $=c^{\prime} d^{* \prime} R_{\mathfrak{p}_{i}^{*}}^{*}=d^{* \prime} R_{\mathfrak{p}^{*}}^{*} \subseteq \mathfrak{p}_{i}^{* \prime n} R_{\mathfrak{p}_{i}^{*}}^{*}=b^{\prime n} R_{\mathfrak{p}_{i}^{*}}^{*}(i=1, \cdots, h)$. Therefore, $r^{*} \in \bigcap_{1}^{h} \mathfrak{p}_{i}^{*(n-1)}$, so by induction $r^{*} \in \mathfrak{p}^{(n-1)} R^{*}$. Thus $c d^{*}=b r^{*} \in \mathfrak{p}^{(n)} R^{*}$, hence $d^{*} \in \mathfrak{p}^{(n)} R^{*}: c R^{*}=\left(\mathfrak{p}^{(n)}: c R\right) R^{*}\left[4\right.$, p. 267] $=\mathfrak{p}^{(n)} R^{*}$, since $c \notin \mathfrak{p}$. 
Thus $\bigcap_{1}^{h} \mathfrak{p}_{i}^{*(n)} \subseteq \mathfrak{p}^{(n)} R^{*}$, and since the opposite inclusion is clear, $\mathfrak{p}^{(n)} R^{*}=\bigcap_{1}^{h} \mathfrak{p}_{i}^{*(n)}$, q.e.d.

THEOREM 1. Let $R$ be a semi-local ring with Jacobson radical $J$, and let $R^{*}$ be the completion of $R$. Assume there is a nonzero-divisor $b$ in $R$ such that $(b R)_{a}=b R$ and $\mathfrak{p} R^{*}$ is semi-prime, for every prime divisor $\mathfrak{p}$ of $b R$. Then $\left(b R^{*}\right)_{a}=b R^{*}$. If $b \in J$, then $R$ is analytically unramified.

Proof. If $b$ is a unit in $R$, then $\left(b R^{*}\right)_{a}=b R^{*}=R^{*}$. Hence assume $b$ is a nonunit in $R$, and let $p_{1}, \cdots, p_{n}$ be the prime divisors of $b R$. Since each $R_{\mathfrak{p}_{\mathfrak{i}}}$ is a discrete Archimedian valuation ring (Lemmas 2 and 4$)$, every $\mathfrak{p}_{i}$-primary ideal is a symbolic power of $\mathfrak{p}_{i}$. Therefore $b R=\bigcap_{1}^{n} \mathfrak{p}_{i}^{\left(e_{i}\right)}$, so $b R^{*}=\bigcap_{1}^{n}\left(\mathfrak{p}^{\left(e_{i}\right)} R^{*}\right)\left[4\right.$, p. 269]. Fix $i$, set $\mathfrak{p}^{(e)}=\mathfrak{p}_{i}^{\left(e_{i}\right)}$, and let $\mathfrak{p}_{1}^{*}, \cdots, \mathfrak{p}_{h}^{*}$ be the prime divisors of $\mathfrak{p} R^{*}$. Then $\mathfrak{p}^{(e)} R^{*}=\bigcap_{1}^{h} \mathfrak{p}_{i}^{*(e)}$ and each $R_{p_{i} *}^{*}$ is normal (Lemma 7). Thus the prime divisors of $b R^{*}$ are the prime divisors of the $\mathfrak{p}_{i} R^{*}(i=1, \cdots, h)$, hence $\left(b R^{*}\right)_{a}=b R^{*}$ (Lemma 4). Therefore, if $b \in J$, then by Corollary $4, R$ is analytically unramified, q.e.d.

Corollary 5. Let $R, R^{*}$ and $b$ be as in Theorem 1 , and let $S^{*}$ be the integral closure of $R^{*}$ in its total quotient ring. If there is an element $v$ in $S^{*}$ such that $b v \in R^{*}$, then $v \in R^{*}$.

Proof. $b v \in b S^{*} \cap R^{*}=\left(b R^{*}\right)_{a}=b R^{*}$, q.e.d.

\section{REFERENCES}

1. M. Nagata, Local rings, Interscience Tracts in Pure and Applied Mathematics, Interscience, New York, 1962.

2. O. Zariski, Analytical irreducibility of normal varieties, Ann. of Math. 49 (1948), $352-361$.

3. O. Zariski and P. Samuel, Commutative algebra, Vol. I, Van Nostrand, Princeton, N. J., 1958.

4. - Commutative algebra, Vol. II, Van Nostrand, Princeton, N. J., 1960.

University of California, Riverside 